\title{
Disentangling the Role of Chain Conformation on the Mechanics of Polymer Tethered Particle Materials
}

\author{
Jiarul Midya, ${ }^{\dagger, \perp} \odot$ Yu Cang, ${ }^{\ddagger, \perp}$ Sergei A. Egorov, ${ }^{\S}$ Krzysztof Matyjaszewski, ${ }^{\# \odot ~ M i c h a e l ~ R . ~ B o c k s t a l l e r, ~}{ }^{\| \odot}$ \\ Arash Nikoubashman, $*,+\infty$ and George Fytas*, \\ ${ }^{\dagger}$ Institute of Physics, Johannes Gutenberg University Mainz, Staudingerweg 7, 55128 Mainz, Germany \\ ${ }^{\ddagger}$ Max Planck Institute for Polymer Research, Ackermannweg 10, 55128, Mainz, Germany \\ ${ }^{\S}$ Department of Chemistry, University of Virginia, McCormick Road, Charlottesville, Virginia 22904-4319, United States \\ ${ }^{\#}$ Chemistry Department, Carnegie Mellon University, 4400 Forbes Avenue, Pittsburgh, Pennsylvania 15213, United States \\ "Department of Materials Science and Engineering, Carnegie Mellon University, 5000 Forbes Avenue, Pittsburgh, Pennsylvania \\ 15213, United States
}

\section{Supporting Information}

ABSTRACT: The linear elastic properties of isotropic materials of polymer tethered nanoparticles (NPs) are evaluated using noncontact Brillouin light spectroscopy. While the mechanical properties of dense brush materials follow predicted trends with NP composition, a surprising increase in elastic moduli is observed in the case of sparsely grafted particle systems at approximately equal NP filling ratio. Complementary molecular dynamics simulations reveal that the stiffening is caused by the coil-like conformations of the grafted chains, which lead to stronger polymer-polymer interactions compared to densely grafted NPs with short chains. Our results point to novel opportunities to enhance the physical properties of composite materials by the strategic design of the "molecular architecture" of constituents to benefit from synergistic effects relating to the organization of the polymer component.

KEYWORDS: Polymer composite, mechanical reinforcement, particle brush, Brillouin light spectroscopy, coarse-grained simulation

\section{Grafting

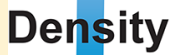

Dense particle brush
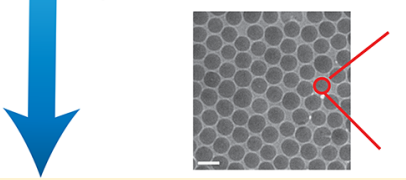
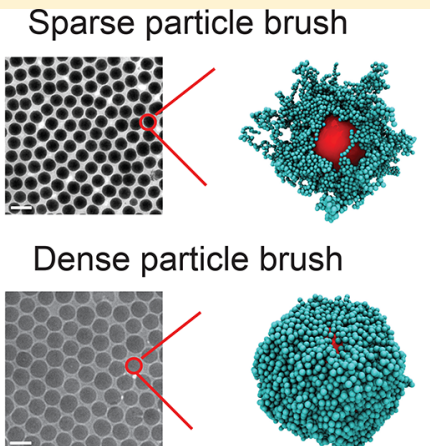

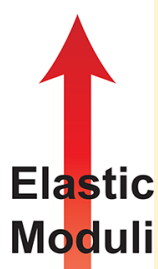

ver the last few decades, extensive studies validated the enhancement of the mechanical properties of polymer composites through the incorporation of nanoparticle (NP) fillers into the polymer matrix. ${ }^{1-3}$ Although the details of this reinforcement in two-component nanocomposite systems remain an open question, experiments have suggested that it originates from the subtle interplay of interactions between polymer and particle constituents and spatial confinement. ${ }^{4-6}$ In the solid (glassy) state, the uniform spatial dispersion of NPs is crucial for attaining optimal mechanical response. However, the inherent chemical incompatibility of the two components renders the fabrication of homogeneous dispersions a formidable task, especially at high NP loading. ${ }^{8-10}$ Brush particles (sometimes referred to as "hairy nanoparticles") that are formed by tethering of polymer chains to the surface of NPs have attracted interest as building blocks for the assembly of "one-component hybrid materials" with unprecedented property combinations. Examples include the synthesis of transparent high refractive index glasses for optical applications, high-breakdown dielectrics, moldable photonic crystals, and phononic crystal materials with robust band gap characteristics. ${ }^{11-15}$
The emergence of new property combinations has been attributed to the effect of steric confinement on the conformation and dynamical properties of tethered chains, and the resulting implications on particle interactions. ${ }^{16}$ Insights into the role of brush architecture on the interaction and relaxation of tethered chains have been provided by studies of the rheological properties and the local and global chain dynamics in particle brush-based materials. ${ }^{10,17-20}$ Collectively, these studies have shown that the effect of steric confinement on the interactions between brush particles sensitively depends on the density and degree of polymerization of tethered chains as well as particle surface curvature. In sufficiently dense-tethered systems, a gradual transition from hard sphere to soft polymer-like interactions has been observed with increasing degree of polymerization. ${ }^{21}$ Glass transition temperature and chain relaxation times were observed to exceed those of corresponding linear polymer analogs, thus indicating that the tethering of chains limits cooperativeness in

Received: February 25, 2019

Revised: March 18, 2019

Published: March 26, 2019 
brush layers $^{17,19}$ and pronounced slowdown of local dynamics at the interface. ${ }^{20}$

As the grafting density of tethered chains is reduced to values of about $0.05 \mathrm{~nm}^{-2}$ (the so-called "sparse" grafting regime), molecular segregation of the tethered chains has been argued to result in directional interaction potentials between individual brush particles. ${ }^{10,11}$ Direct evidence for the "polarization" of particle interactions is the formation of anisotropic superstructures in sparse particle brush systems. ${ }^{10}$ Interestingly, tensile stress-strain experiments have revealed that the formation of anisotropic superstructures gives rise to significant enhancement of the mechanical behavior of the resulting hybrid materials. ${ }^{22}$ Due to limited experiments on the mechanical properties of one-component systems, the role of graft architecture is still unclear. The elastic modulus of sparsely polystyrene (PS) grafted NP films, measured by thin film buckling techniques, was found to be independent of the graft molecular weight $\left(30 \mathrm{kDa}<M_{\mathrm{W}}<370 \mathrm{kDa}\right) .{ }^{23}$ Molecular dynamics (MD) simulations predicted an increase of fracture toughness with increasing chain length, implying that entanglements affect interparticle interactions. ${ }^{24,25}$ These findings illustrate the opportunities to design hybrid material properties that are facilitated by a better understanding of the governing parameters, which control interactions in brush particle systems. However, no study has been yet able to quantitatively relate macroscopic response characteristics to microstructural features such as the distribution of tethers within the brush particle assemblies. The elucidation of the role of polymer canopies on the elastic (bulk and shear) moduli of polymer tethered particle materials is thus an important requisite to further advance the opportunities presented by brush particle materials. ${ }^{26}$

In this contribution we shed light onto this question by performing noncontact and nondestructive mechanical characterization using Brillouin light spectroscopy (BLS). BLS is a powerful optical technique to probe the acoustic phonon propagation at gigahertz frequencies and hence access the elastic characteristics of materials on a submicrometer scale. We complement the BLS experiments with microscopically resolved MD simulations, ${ }^{27-29}$ which capture the conformational details of the grafted chains. We employ a coarse-grained model where the silica core is represented by a smooth sphere with randomly distributed grafting points on its surface, while the grafted PS chains are modeled using a generic bead-spring model. ${ }^{30}$ The polymer beads have a 20 times smaller diameter than the cores, and the interaction strengths between the different compounds were matched to the experimental Hamaker constants. ${ }^{31}$ Such a coarse-grained description is computationally more tractable than an atomistic model, and it also allows us to derive general trends which are independent of the specific polymer and NP chemistry. Finally, we also employ a much more coarse-grained model of sterically stabilized NPs and combine it with the Zwanzig-Mountain relation and integral equation theory to compute the highfrequency shear modulus. The details of the experimental and computational methods are provided in the Supporting Information.

Our experiments reveal that, for composites with equal inorganic content, the longitudinal and shear modulus increase by about $35 \%$ as the grafting density is decreased. This finding is in stark contrast to mean-field calculations, which predict the same moduli for both materials. Previous experiments and finite element analysis have hypothesized that such reinforce- ment could originate from the formation of a thin $(2-3 \mathrm{~nm}$ wide) interfacial layer with significantly higher modulus than that of the neat polymer. ${ }^{4,5,26}$ However, this interpretation is not supported at all by our MD simulations. Instead, we find that the increase of the bulk modulus originates from the overlap of the polymer grafts, which is more pronounced for the sparsely grafted NPs with long chains. At roughly equal chain length but different grafting density, the sparsely grafted NPs have a stronger polymer-polymer contribution to the elastic modulus, due to the enhanced entanglement between chains from different NPs. However, this effect is only secondary, as the major difference in the elastic moduli stems from the core-polymer contribution, which is weaker in the densely grafted systems and their lower NP loading. Thus, the conformation of the tethered polymers plays a crucial role for the elastic properties of the nanocomposite.

The one-component nanocomposite systems used in this study consist of PS grafted silica particles with varying grafting density (number of chains per area), $\sigma$, and degree of polymerization (DP), $N$, below and above the entanglement value of bulk PS. The brush particle systems are synthesized via surface-initiated atom transfer radical polymerization of PS from identical silica cores with radius $R=57 \pm 3 \mathrm{~nm}$ and coded according to their DP; PS is in the amorphous glassy state at $T=294 \mathrm{~K}^{32}$ (See details in the material part of the Supporting Information.) Figure 1 presents the brush thickness, $h$, as determined by the distance between silica cores from transmission electron microscopy (TEM) images. For the high grafting density $\left(\sigma \approx 0.5\right.$ chains $\left./ \mathrm{nm}^{2}\right)$, the double logarithmic plot clearly displays a stronger increase of brush thickness $h$ with $N$ than expected for a Gaussian chain with the same degree of polymerization. The extended chain conformation is represented by the scaling relation $h \propto N^{0.7}$ in the low- $N$ regime, whereas a crossover to an ideal coil, i.e., $h \propto$ $N^{1 / 2}$, is observed for the densely tethered materials above $N \approx$ 1000. The relations between brush height and $\sigma$ and/or $N$ mirror the conformation of the polymer grafts and further indicate the extent of chain interpenetration between neighbor particles. With decreasing grafting density (from $0.61 \mathrm{~nm}^{-2}$ (DP 100) to $0.08 \mathrm{~nm}^{-2}$ (DP1170)), the tethered NPs assume more compact dimensions as it is clearly revealed in the case of DP1170. A more detailed analysis of the polymer conformations is provided further below, where we show the distributions of polymer end-to-end lengths and compare them to the corresponding pure polymer systems.

The sparsely tethered DP1170 system assumes a 5 times smaller brush thickness than the densely tethered DP1300 with comparable $N$. Instead, DP1170 displays almost the same brush thickness as the densely tethered DP100 material, and hence both possess similar PS volume fractions, $\phi_{\mathrm{PS}} \approx 0.48$. This composition was chosen since effective medium theory would predict comparable elasticities in both DP100 and DP1170 materials due to their similar average $\phi_{\mathrm{PS}}$. Concurrently, low $\sigma$ and high $N$ should then facilitate chain interpenetration between polymer canopies as has been proposed. ${ }^{17,34}$ We found similar NP loading in our MD simulations, as evidenced by the qualitative comparison of the NP distributions in Figure $1 \mathrm{~b}$. Note that the (hexagonal) ordering of NPs in the experimental TEM micrographs originates most likely from casting the brush particles to monolayer films for imaging, whereas the simulation snapshots have been taken from bulk systems. The experimental BLS measurements were performed using thick films, and thus the 
(a)

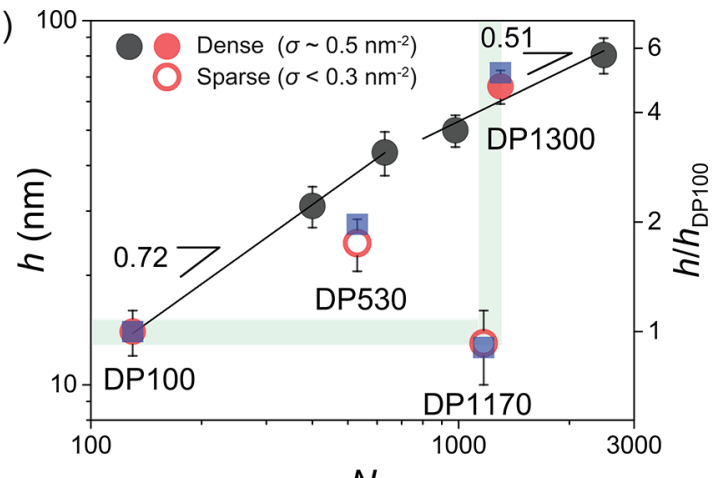

(b)

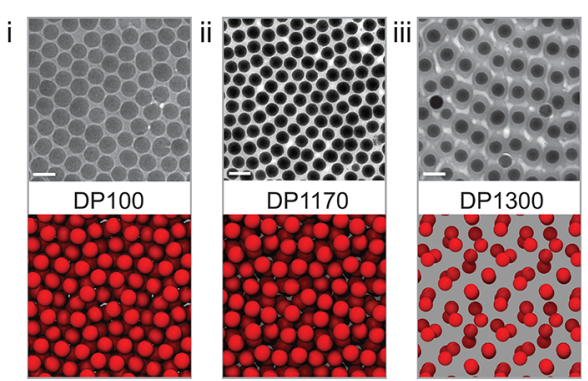

(c)

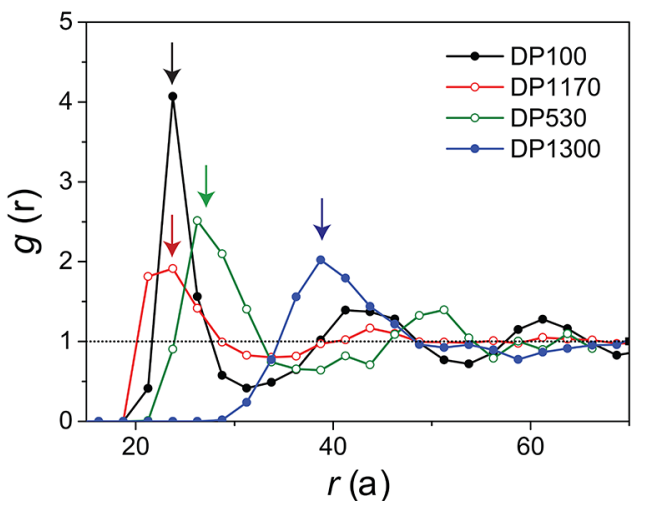

Figure 1. Tethered chain size in polymer grafted colloids. (a) Thickness of grafted PS, $h$, for densely (solid circles) and sparsely (open circles) grafted NPs as a function of the PS degree of polymerization, $N$. The particle systems primarily discussed in this work and in the ref 15 are labeled with red and black circles, respectively. The right axis shows the normalized thickness, $h / h_{\mathrm{DP} 100}$, relative to its value for the particle brush with $N=100$. Results from MD simulations are shown by blue squares in this representation. The two shaded stripes indicate two systems with almost identical PS volume fraction (DP100, DP1170) and similar PS molecular weight (DP1170, DP1300), respectively. (b) TEM images of three ordered one-component polymer nanocomposite monolayer films with different $N$ and $\sigma$ (upper panel), and corresponding simulation snapshots (lower panel). The "layering" of brush particles that is observed in micrograph (iii) is attributed to in-plane stresses that arise during the transfer of films. This effect is absent in bulk samples that are evaluated by BLS. ${ }^{15}$ The simulation snapshots have been duplicated once in both the horizontal and vertical direction to better match the experimental scale. Further, the polymer brush has not been drawn here for clarity. All simulation snapshots have been rendered using Visual Molecular Dynamics. ${ }^{33}$ (c) Radial pair distribution functions, $g(r)$, for four nanocomposites computed from MD simulations, as indicated with $r$ denoting the radial distance between NP centers, in the units of monomer diameter, $a$. The colored arrows indicate the brush height of the four grafted PS particles extracted from the corresponding $g(r)$. apparent ordering is not relevant for the interpretation of the elastic moduli data.

From the MD trajectories, we computed the radial pair distribution function, $g(r)$, between NPs (Figure 1c). From these data, we extracted the brush thickness, $h$, by identifying the nearest neighbor distance between the NPs. The pronounced oscillations of the $g(r)$ data indicate that the NPs exhibit long-range ordering, with the DP100 system being the most ordered one. When normalizing the computed brush thickness, $h$, by the value obtained for DP100, $h_{\mathrm{DP} 100}$, we find excellent agreement with the experiments, as shown in Figure 1a. Note that both experimental and simulation results confirm the absence of anisotropic particle superstructures that have been reported for very sparse systems in previous reports. ${ }^{8,10}$

Having established the good agreement between the structures from experiments and $\mathrm{MD}$ simulations for the four systems in Figure 1, we turn now to their elastic moduli. As a noncontact and noninvasive technique, BLS becomes a powerful and unique tool to analyze the elastic mechanical properties compared to invasive atomic force microscopy (AFM) indentation. The effective medium sound velocities, $c_{l(t)}=2 \pi f_{l(t)} / q$ can be determined from the linear dispersion, $f_{l(t)}(q)$ obtained from the polarized (depolarized) BLS spectra at a sufficiently low wave vector, $q$. The subscript $l(t)$ denotes the longitudinal (transverse) polarization of phonons (Supporting Information). Figure 2a shows the linear dispersion, $f_{l(t)}(q)$ in DP1170, where the frequencies $f_{l(t)}$ are estimated from the Lorentzian representation of the polarized (VV) and depolarized (VH) BLS spectra shown in the inset of Figure 2a. The longitudinal $(M)$ and shear $(G)$ moduli, are computed by assuming a linear interpolation of the effective medium density, $\rho$, between the two-component densities (see details in Table S1), i.e., $M=\rho c_{l}^{2}$ and $G=\rho c_{t}^{2}$. Figure $2 \mathrm{~b}$ depicts the values of $M$ and $G$ in eight polymer tethered nanocomposite materials at $T=294 \mathrm{~K}$ below the glass transition of PS $\left(T_{\mathrm{g}}=373 \mathrm{~K}\right)$. Note that the elastic (high frequency) moduli become local properties in the glassy state and hence are not sensitive to the inherently metastable glass. ${ }^{34}$ The volume fraction for the previously reported and the present systems has been estimated within the indicated errors by different methods as reported elsewhere. ${ }^{34}$ Both elastic moduli show significant increase with particle loading. This trend of densely grafted particle systems can be compared with results from effective medium theory (such as Wood's law) using the parameters for PS $\left(\rho=1050 \mathrm{~kg} / \mathrm{m}^{3}, c_{\mathrm{L}}=2350 \mathrm{~m} / \mathrm{s}\right.$, and $\left.c_{\mathrm{T}}=1210 \mathrm{~m} / \mathrm{s}\right)$ and for porous silica $\left(\rho=1850 \mathrm{~kg} / \mathrm{m}^{3}, c_{\mathrm{L}}=4910 \mathrm{~m} / \mathrm{s}\right.$, and $c_{\mathrm{T}}=$ $3090 \mathrm{~m} / \mathrm{s}$ ). The generally accepted effective medium expression $^{35}$ for two-component systems fails to represent the $M$ and $G$ of our systems (Figure S6). The elastic moduli for the densely grafted systems, however, follow Wood's law, whereas the more sparsely grafted particle systems exhibit up to $35 \%$ higher moduli than predicted by Wood's law (Figure $2 b)$.

The compression modulus, $K=M-(4 / 3) G$, of DP1170 is also higher (about 20\%) than in DP100, as shown in the normalized plot of Figure 2c. To support the experimental findings and to elucidate the origin of the surprising strengthening effect, we computed the high-frequency shear modulus $G_{\infty}$ using the Zwanzig-Mountain relation ${ }^{36}$ and the compression modulus through MD simulations. The effective interaction potential ${ }^{37}$ for the calculation of $G_{\infty}$ is based on modeling the grafted chains as a spherical layer of uniform effective density comprised of the polymeric segments. This 

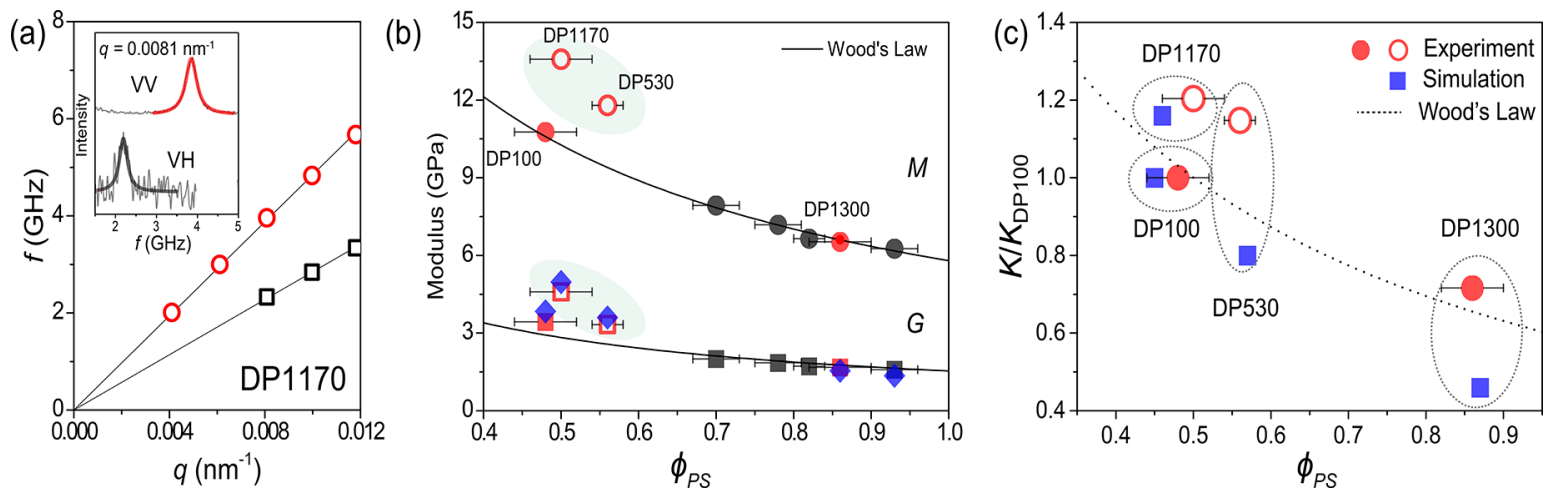

Figure 2. Composition dependence of the elastic moduli in polymer grafted particle films. (a) Dispersion relation of longitudinal (red circles) and transverse phonons (black squares) of DP1170 in the low- $q$ regime. Inset: exemplary VV polarized and VH depolarized BLS spectra fitted by Lorentzian functions at $q=0.0081 \mathrm{~nm}^{-1}$. (b) Longitudinal, $M$ (circles), and shear, $G$ (squares), elastic moduli for various PS tethered silica films with different PS volume fraction for densely (solid circles and squares) and sparsely (open circles and squares in the shaded areas) grafted particle systems. The theoretical values of the shear moduli in five nanocomposite films are indicated by blue solid rhombs. The representation by the effective medium Wood's law is shown by solid lines. (c) Normalized compression modulus relative to the value in the DP100 system, obtained from BLS and MD simulations for four systems, as indicated. The Wood's law prediction is shown by the dashed line.

density is simply calculated from the experimental $N, \sigma$, and $h$ (Figure 1a) leading to the total number of segments and the volume of the spherical shell. The effective shell density is therefore higher, and hence the interaction potential is stronger in the sparsely tethered DP1170 than in the brush-like DP100. Accordingly, ${ }^{36}$ DP1170 exhibits a higher shear modulus (blue solid rhombs) in agreement with the BLS experimental values of Figure $2 \mathrm{~b}$. Even though this effective model is fundamentally different from the microscopic MD simulations (e.g., no chain interdigitation/entanglements are included), it accurately reproduces the experimental trends in the present (Figure 2b) and similar systems. ${ }^{38}$ Since the elastic shear modulus (no viscoelastic effects) primarily depends on the local segment density, topological effects are not relevant. ${ }^{39}$ In any case, the main advantage of this effective interaction model is its extreme computational simplicity, and it is gratifying that it still captures the experimental trends (Figure 2b). This good agreement between the experimental measurements and the computed values for $G_{\infty}$ further rationalizes the deviations from Wood's law prediction.

To elucidate the significant deviations of the elastic moduli from effective medium laws, we considered the microscopic details of the polymer conformations provided by the MD simulations. To this end, Figure 3 shows simulation snapshots of single grafted NPs in the polymer nanocomposite (note that these snapshots have been obtained from the bulk materials, not from simulations of isolated NPs). We focus first on the DP100 and DP1170 systems, which exhibit similar brush thickness, and hence similar filling fraction, but significantly different elastic properties. From Figure 3a, it is clear that the high grafting density of DP100 leads to an even brush layer of strongly stretched chains surrounding the NP core. For DP1170, in contrast, the low grafting density leaves large portions of the NP core bare and thus accessible to other NPs. The simulation snapshot in Figure $3 \mathrm{~b}$ further reveals coil-like polymer conformations, similar to the ones observed in the bulk (see Figure 4a). The situation for DP1300 is qualitatively similar to the DP100 case, with a thick polymer shell also obscuring the NP core, albeit the grafted chains are more coiled for DP1300 (Figure 4a). It should be further noted that the polymer layer is not perfectly spherically symmetric, since some of the chains have to stretch to fill the interstitial voids between NPs, as also suggested by TEM. ${ }^{15}$ The structure of the DP530 NPs falls in between the DP100 and DP1300 cases, thus accounting for the intermediate compression modulus computed from the MD simulations (cf. Figure 2c).

The thickness and density of the brush layer as well as the degree of interdigitation between neighboring polymer coronas can be quantified through the radial monomer density distribution around the NP core, $\rho(r)$, presented in Figure 3. In all cases, there is a noticeable depletion of $\rho$ followed by a slight surplus of $\rho$ close to the NP surface, as expected for the layering of hard particles near a hard surface. ${ }^{40}$ The structuring near the NP surface is, however, rather subtle and decays completely after few monomers. Figure 3 also shows the breakdown into contributions from polymers grafted to the same core, $\rho_{\mathrm{s}}$, and from surrounding polymers grafted to other cores, $\rho_{\mathrm{o}}$. For the densely grafted NPs DP100, DP530, and DP1300, the first layer around an NP is completely occupied by its own grafted chains, and $\rho_{\mathrm{s}}$ then gradually decays to zero as a distance $r \approx h$ is approached. For DP1170, however, a large fraction of polymers from other NPs penetrates the brush layer of the NP, leading to considerable overlap and interdigitation of the grafted polymer chains. The overlap of polymer brushes can be quantified through the interpenetration parameter $\delta$ (see the Supporting Information for details), which has a significantly higher value for DP1170 $(\delta=120)$ compared to DP100 $(\delta=39)$ and DP530 $(\delta=92)$. For DP1300, the value is largest $(\delta=150)$, but here the brush overlap occurs far from the NP cores (see Figure 3d).

The MD simulations also facilitate a detailed analysis of the chain conformations, expressed by the probability distribution of the end-to-end distance, $R_{\mathrm{e}}$, normalized by the chain contour length, $L_{\mathcal{c}}$, plotted in Figure $4 a$. In this graph, we have also included the corresponding distributions for bulk PS chains as a point of reference. These distributions clearly indicate that the DP100 chains are strongly stretched due to their high grafting density, whereas the DP1170 chains assume coil-like conformations much closer to their bulk analog. This behavior is also reflected by the average end-to-end distances of the polymers, $\left\langle R_{\mathrm{e}}\right\rangle$, where we observed increases by $38 \%$ (DP100), 7\% (DP1170), 24\% (DP530), and 57\% (DP1300), relative to the corresponding values for the pure polymer systems. Moreover, we found a local stiffening of polymer 
(a)
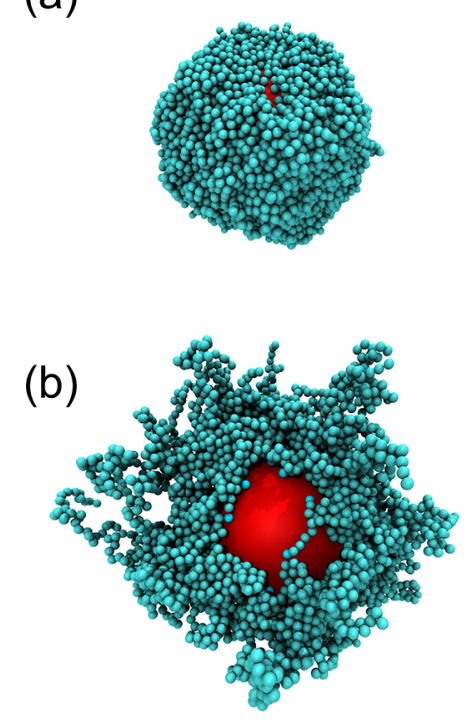

(c)

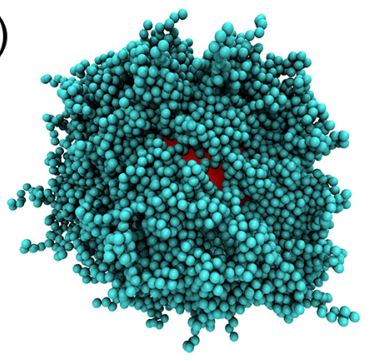

(d)

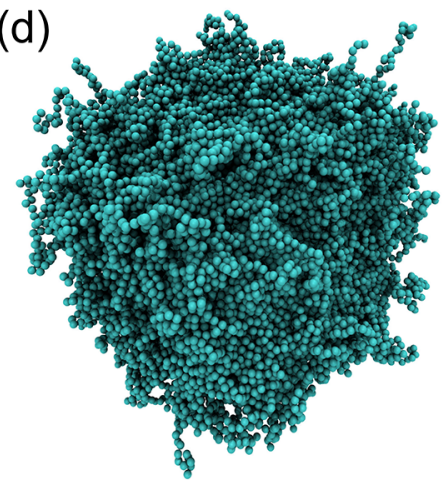

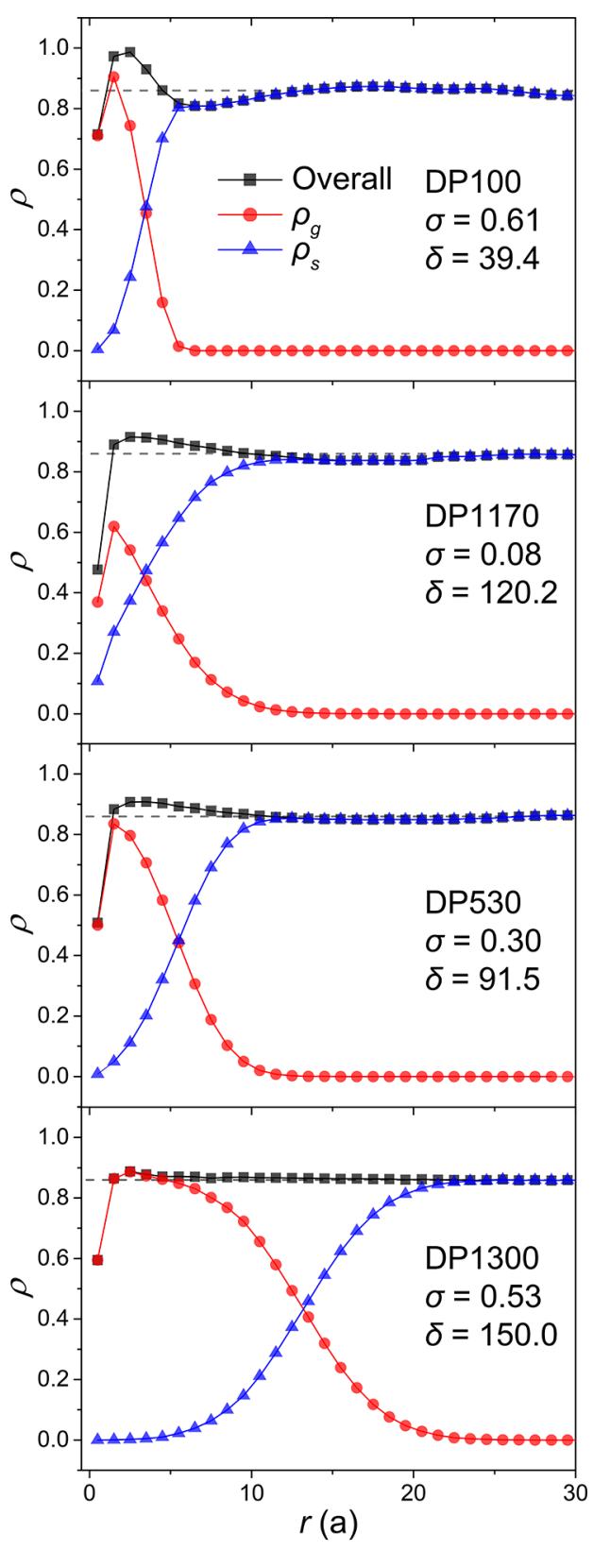

Figure 3. Tethered chain architecture in polymer grafted nanoparticles. Left panel: Simulation snapshots of a single nanoparticle in the nanocomposite for (a) DP100, (b) DP1170, (c) DP530, and (d) DP1300. Right panel: Monomer density, $\rho$, as a function of distance to the core surface, $r$, in the unit of monomer diameter, $a$. Contributions from chains grafted to the same core $\left(\rho_{s}\right)$ are shown as red circles, from other nanoparticles $\left(\rho_{\mathrm{o}}\right)$ as blue triangles, and the total density is shown as black squares. The dashed horizontal line indicates the overall monomer density in the system.

segments close to the surface of the rigid NP cores (see the Supporting Information). This effect was most pronounced for DP100 (highest grafting density), where the local persistence length increased by a factor of roughly 2 for segments directly adjacent to the NP core. In contrast, for DP1170 (lowest grafting density) almost no local chain stiffening could be observed. In all cases, the chain persistence length decayed after few segments to the bulk value.

The compression modulus in Figure $2 c$ decreases with PS volume fraction (or equivalently, brush thickness, $h$ ) for the high grafting density particle brush systems in both experiments and MD simulations. This behavior appears to contradict recent AFM-indentation results for which the Young modulus of high grafting density NP films increased with increasing $N^{41,42}$ The MD simulations also capture the higher compression modulus in the sparsely grafted DP1170 sample than in the densely grafted DP100 systems at similar $\phi_{\mathrm{PS}} \approx$ 0.48. To elucidate the mechanism of this enhancement as well as the drop of $K$ with $N$ for the high grafting density NPs, we determined the individual contributions to the compression modulus $K$, which is possible in the MD simulations (see the Supporting Information for details). Figure $4 \mathrm{~b}$ shows the core-core $\left(K_{c-c}\right)$, the core-polymer $\left(K_{c-p}\right)$, and the polymer-polymer $\left(K_{\mathrm{p}-\mathrm{p}}\right)$ part of $K$, revealing several key trends: (i) Core-core contributions are negligible for the investigated samples due to their rather high PS volume 

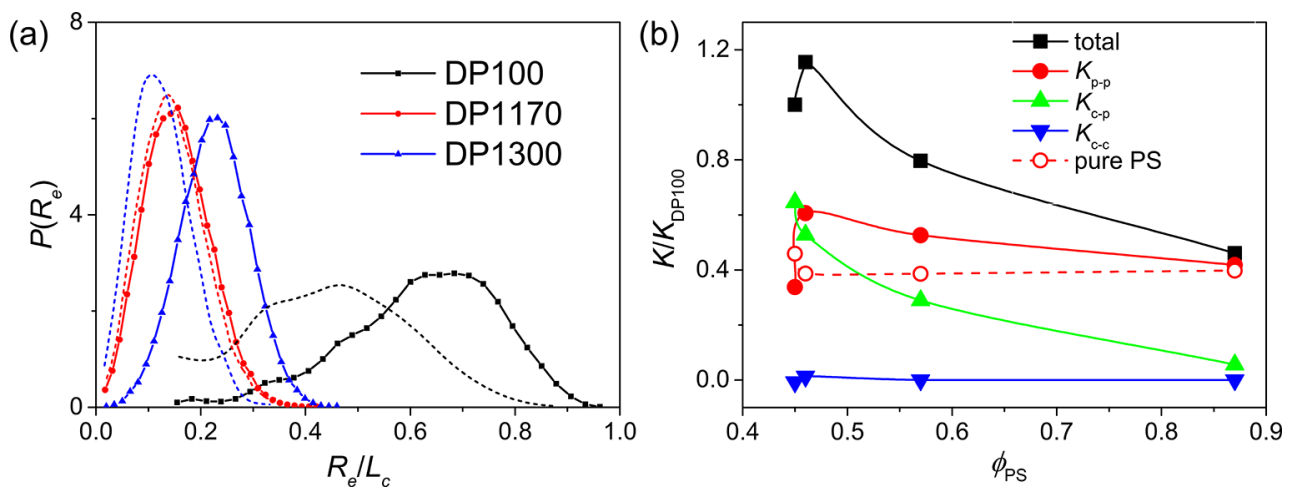

Figure 4. (a) Plot of end-to-end distribution, $P\left(R_{\mathrm{e}}\right)$, of polymer chains grafted on the nanoparticles (solid lines) and the corresponding PS chains in bulk (dotted lines), as a function of $R_{\mathrm{e}} / L_{\mathrm{c}}$ (with contour length $L_{\mathrm{c}}$ ). (b) Contributions to the compression modulus from MD simulations. The dashed red line indicates the compression modulus of bulk PS. All data are normalized relative to the compression modulus of DP100.

fraction, $\phi_{\mathrm{PS}}$, which effectively shields the cores from each other. Further, $K_{c-c}$ is almost identical for all samples, with a shallow maximum for DP1170, consistent with the "bald spot" of the NP shown in Figure 3b. (ii) The core-polymer contribution to the compression modulus decreases monotonically with increasing $\phi_{\mathrm{PS}}$ (or with increasing brush thickness $h$ ), as it becomes more and more difficult for the grafted chains to penetrate the polymer layer around other NPs. (iii) The polymer-polymer contribution is nonmonotonic with respect to $\phi_{\mathrm{PS}}$ and has a maximum for DP1170 and a minimum for DP100. This behavior originates from interdigitation and entanglement of the polymer grafts (see discussion below).

Thus, the significant difference between $K_{\mathrm{DP} 100}$ and $K_{\mathrm{DP} 1170}$ stems predominantly from the polymer-polymer contribution, $K_{\mathrm{p}-\mathrm{p}}$, which is considerably larger for the DP1170 sample due to the strong overlap and interdigitation of the polymer brushes. This effect cannot be captured by a mean-field description of the composite, due to the erroneous assumption that $K_{\mathrm{p}-\mathrm{p}}$ is the same for all samples, which is clearly not the case here. Instead, the additional constraints imposed by the NP cores lead to strong deviations of $K_{\mathrm{p}-\mathrm{p}}$ compared to the values determined from bulk simulations of the pure polymer (see dashed red line in Figure 4b). Further, it is clear from the data shown in Figure $4 \mathrm{~b}$ that the decrease of $K$ with increasing $\phi_{\mathrm{PS}}$ is dominated by the strongly decreasing core-polymer contribution, $K_{\mathrm{c}-\mathrm{p}}$.

Figure $4 \mathrm{~b}$ also shows that $K_{\mathrm{p}-\mathrm{p}}$ is slightly smaller for the densely grafted DP1300 systems compared to the sparse DP1170 NPs at roughly the same degree of polymerization. To understand this behavior, we determined the degree of chain entanglements by computing the average number of double kinks per chain, $\langle Z\rangle$, using the $\mathrm{Zl}$ algorithm (see the Supporting Information for details). ${ }^{43,44}$ For the total number, we find $\left\langle Z_{\text {tot }}\right\rangle=0.0013$ for DP100, $\left\langle Z_{\text {tot }}\right\rangle=0.13$ for DP530, $\left\langle Z_{\text {tot }}\right\rangle=0.24$ for DP1170, and $\left\langle Z_{\text {tot }}\right\rangle=0.32$ for DP1300. As expected, the number of entanglements increases with the degree of polymerization. More interesting is the breakdown of $\left\langle Z_{\text {tot }}\right\rangle$ into contributions from chains grafted to the same NP, $\left\langle Z_{\mathrm{s}}\right\rangle$, and from chains grafted to other NPs, $\left\langle Z_{\mathrm{o}}\right\rangle=\left\langle Z_{\text {tot }}\right\rangle-$ $\left\langle Z_{\mathrm{s}}\right\rangle^{25}$ Here, we find $\left\langle Z_{\mathrm{o}}\right\rangle=0.13$ for DP1170, and $\left\langle Z_{\mathrm{o}}\right\rangle=$ 0.082 for DP1300. For DP100 and DP530 inter-NP chain entanglements are negligible, as expected from the rather short length of the polymer grafts. Interestingly, DP1170 exhibits significantly more inter-NP chain entanglements than DP1300, although the total number of entanglements is smaller for the sparsely grafted NPs. These inter-NP entanglements provide additional stiffness to the composite, as evidenced by the slight increase of $K_{\mathrm{p}-\mathrm{p}}$ shown in Figure $4 \mathrm{~b}$. Further note that these differences in the polymer-polymer interactions originate entirely from grafting the chains to a NP, which dramatically reduces the conformational degrees of freedom of the polymers. For the pure polymer systems of DP1170 and DP1300, the elastic properties and the entanglement statistics are almost identical.

Conclusions. We utilized Brillouin light spectroscopy, a noncontact and nondestructive technique, to measure the longitudinal and transverse sound velocities and obtain the elastic engineering moduli (Young's, bulk, and shear modulus) of isotropic materials of polystyrene tethered (silica) nanoparticles (NPs) (core radius $57 \pm 3 \mathrm{~nm}$ ) with different length and density of grafted chains. All elastic moduli of the dense brush materials $\left(\approx 0.5\right.$ chains $\left./ \mathrm{nm}^{2}\right)$ increase with NP filling ratio, following predictions from effective medium theory. In contrast, both the longitudinal and shear modulus increase significantly (by about 30\%) in the case of a sparsely grafted (0.08 chains $\left./ \mathrm{nm}^{2}\right) \mathrm{NP}$ system at approximately equal NP filling $\left(\phi_{\mathrm{PS}} \approx 0.48\right)$.

Microscopically resolved molecular dynamics simulations confirm the experimentally observed trends, and reveal that the stiffening at high NP loading originates from the overlap of the polymer brushes, which is much more pronounced for the sparsely grafted NPs with long chains compared to the densely grafted NPs with short chains. Further, our simulations show that, for equal chain length but different grafting density, the polymer-polymer contribution to the compression modulus is enhanced for the sparsely grafted systems due to the increased number of inter-NP chain entanglements. This effect is, however, only secondary, as the main difference in compression modulus originates from the core-polymer contribution, which is significantly smaller for the densely grafted NP system due to the lower NP loading. The experimentally observed trend of the high-frequency shear modulus with grafting density and polymer graft length was well captured by a simple coarse-grained model of polymer tethered NPs. Despite the simplicity of the model, the good agreement indicates that the local segment density largely determines the shear modulus $G_{\infty}$. To our knowledge this is the first observation of molecular segregation effects giving rise to enhanced mechanical properties in isotropic brush particle systems. This offers novel opportunities for the design of hybrid material solutions combining enhanced mechanical 
properties with overall macroscopically isotropic response that could be relevant, for example, for optical applications.

\section{ASSOCIATED CONTENT}

\section{S Supporting Information}

The Supporting Information is available free of charge on the ACS Publications website at DOI: 10.1021/acs.nanolett.9b00817.

Experimental details of sample preparation and Brillouin light spectroscopy (including a table of parameters for the PS-tethererd Si particles), description of simulation model and methods (including figures of polymer structures, a table of simulation data, and plots of $E, P$, and $R_{\mathrm{g}}$ vs MD steps and of $P$ vs $V$ ), additional simulation results (including bond-bond autocorrelation function, and persistence length vs bond number), and a schematic of the Rabani model (PDF)

\section{AUTHOR INFORMATION}

\section{Corresponding Authors}

*E-mail: anikouba@uni-mainz.de.

*E-mail: fytas@mpip-mainz.mpg.de.

\section{ORCID $\odot$}

Jiarul Midya: 0000-0003-1695-9059

Krzysztof Matyjaszewski: 0000-0003-1960-3402

Michael R. Bockstaller: 0000-0001-9046-9539

Arash Nikoubashman: 0000-0003-0563-825X

\section{Author Contributions}

${ }^{\perp}$ J. Midya and Y. Cang contributed equally to this work.

\section{Notes}

The authors declare no competing financial interest.

\section{ACKNOWLEDGMENTS}

Y. Cang and G. Fytas acknowledge the financial support by ERC AdG SmartPhon No. 694977. A. Nikoubashman and J. Midya acknowledge funding from the German Research Foundation (DFG) through project NI 1487/2. Further, J. Midya received funding by the Impulsfund of RhinelandPalatinate (Germany). M. R. Bockstaller acknowledges support by the National Science Foundation via grant CMMI-1663305. We thank M. Kröger for providing assistance with the Z1 algorithm. The authors gratefully acknowledge the computing time granted on the supercomputer Mogon at Johannes Gutenberg University Mainz (hpc.uni-mainz.de).

\section{REFERENCES}

(1) Rafiee, M. A.; Rafiee, J.; Wang, Z.; Song, H.; Yu, Z.-Z.; Koratkar, N. Enhanced Mechanical Properties of Nanocomposites at Low Graphene Content. ACS Nano 2009, 3 (12), 3884-3890.

(2) Kamal, M. R.; Khoshkava, V. Effect of cellulose nanocrystals (CNC) on rheological and mechanical properties and crystallization behavior of PLA/CNC nanocomposites. Carbohydr. Polym. 2015, 123, 105-114.

(3) De France, K. J.; Chan, K. J. W.; Cranston, E. D.; Hoare, T. Enhanced Mechanical Properties in Cellulose Nanocrystal-Poly(oligoethylene glycol methacrylate) Injectable Nanocomposite Hydrogels through Control of Physical and Chemical Cross-Linking. Biomacromolecules 2016, 17 (2), 649-660.

(4) Cheng, S.; Bocharova, V.; Belianinov, A.; Xiong, S.; Kisliuk, A.; Somnath, S.; Holt, A. P.; Ovchinnikova, O. S.; Jesse, S.; Martin, H.; Etampawala, T.; Dadmun, M.; Sokolov, A. P. Unraveling the
Mechanism of Nanoscale Mechanical Reinforcement in Glassy Polymer Nanocomposites. Nano Lett. 2016, 16 (6), 3630-3637.

(5) Gong, S.; Chen, Q.; Moll, J. F.; Kumar, S. K.; Colby, R. H. Segmental Dynamics of Polymer Melts with Spherical Nanoparticles. ACS Macro Lett. 2014, 3 (8), 773-777.

(6) Ciprari, D.; Jacob, K.; Tannenbaum, R. Characterization of Polymer Nanocomposite Interphase and Its Impact on Mechanical Properties. Macromolecules 2006, 39 (19), 6565-6573.

(7) Maillard, D.; Kumar, S. K.; Fragneaud, B.; Kysar, J. W.; Rungta, A.; Benicewicz, B. C.; Deng, H.; Brinson, L. C.; Douglas, J. F. Mechanical Properties of Thin Glassy Polymer Films Filled with Spherical Polymer-Grafted Nanoparticles. Nano Lett. 2012, 12 (8), 3909-3914.

(8) Kumar, S. K.; Jouault, N.; Benicewicz, B.; Neely, T. Nanocomposites with polymer grafted nanoparticles. Macromolecules 2013, 46 (9), 3199-3214.

(9) Jancar, J.; Douglas, J. F.; Starr, F. W.; Kumar, S. K.; Cassagnau, P.; Lesser, A. J.; Sternstein, S. S.; Buehler, M. J. Current issues in research on structure-property relationships in polymer nanocomposites. Polymer 2010, 51 (15), 3321-3343.

(10) Akcora, P.; Liu, H.; Kumar, S. K.; Moll, J.; Li, Y.; Benicewicz, B. C.; Schadler, L. S.; Acehan, D.; Panagiotopoulos, A. Z.; Pryamitsyn, V.; Ganesan, V.; Ilavsky, J.; Thiyagarajan, P.; Colby, R. H.; Douglas, J. F. Anisotropic self-assembly of spherical polymer-grafted nanoparticles. Nat. Mater. 2009, 8, 354-359.

(11) Li, Y.; Tao, P.; Viswanath, A.; Benicewicz, B. C.; Schadler, L. S. Bimodal surface ligand engineering: the key to tunable nanocomposites. Langmuir 2013, 29 (4), 1211-1220.

(12) Wang, Z.; Lu, Z.; Mahoney, C.; Yan, J.; Ferebee, R.; Luo, D.; Matyjaszewski, K.; Bockstaller, M. R. Transparent and High Refractive Index Thermoplastic Polymer Glasses Using Evaporative Ligand Exchange of Hybrid Particle Fillers. ACS Appl. Mater. Interfaces 2017, 9 (8), 7515-7522.

(13) Tchoul, M. N.; Fillery, S. P.; Koerner, H.; Drummy, L. F.; Oyerokun, F. T.; Mirau, P. A.; Durstock, M. F.; Vaia, R. A. Assemblies of Titanium Dioxide-Polystyrene Hybrid Nanoparticles for Dielectric Applications. Chem. Mater. 2010, 22 (5), 1749-1759.

(14) Grabowski, C. A.; Koerner, H.; Meth, J. S.; Dang, A.; Hui, C. M.; Matyjaszewski, K.; Bockstaller, M. R.; Durstock, M. F.; Vaia, R. A. Performance of dielectric nanocomposites: matrix-free, hairy nanoparticle assemblies and amorphous polymer-nanoparticle blends. ACS Appl. Mater. Interfaces 2014, 6 (23), 21500-21509.

(15) Alonso-Redondo, E.; Schmitt, M.; Urbach, Z.; Hui, C. M.; Sainidou, R.; Rembert, P.; Matyjaszewski, K.; Bockstaller, M. R.; Fytas, G. A new class of tunable hypersonic phononic crystals based on polymer-tethered colloids. Nat. Commun. 2015, 6, 8309.

(16) Fernandes, N. J.; Koerner, H.; Giannelis, E. P.; Vaia, R. A. Hairy nanoparticle assemblies as one-component functional polymer nanocomposites: opportunities and challenges. MRS Commun. 2013, 3 (1), 13-29.

(17) Kim, S. A.; Mangal, R.; Archer, L. A. Relaxation Dynamics of Nanoparticle-Tethered Polymer Chains. Macromolecules 2015, 48 (17), 6280-6293.

(18) Askar, S.; Li, L.; Torkelson, J. M. Polystyrene-Grafted Silica Nanoparticles: Investigating the Molecular Weight Dependence of Glass Transition and Fragility Behavior. Macromolecules 2017, 50 (4), $1589-1598$.

(19) Koerner, H.; Opsitnick, E.; Grabowski, C. A.; Drummy, L. F.; Hsiao, M.-S.; Che, J.; Pike, M.; Person, V.; Bockstaller, M. R.; Meth, J. S.; Vaia, R. A. Physical aging and glass transition of hairy nanoparticle assemblies. J. Polym. Sci., Part B: Polym. Phys. 2016, 54 (2), 319-330.

(20) Holt, A. P.; Bocharova, V.; Cheng, S.; Kisliuk, A. M.; White, B. T.; Saito, T.; Uhrig, D.; Mahalik, J. P.; Kumar, R.; Imel, A. E.; Etampawala, T.; Martin, H.; Sikes, N.; Sumpter, B. G.; Dadmun, M. D.; Sokolov, A. P. Controlling Interfacial Dynamics: Covalent Bonding versus Physical Adsorption in Polymer Nanocomposites. ACS Nano 2016, 10 (7), 6843-6852.

(21) Ohno, K.; Morinaga, T.; Takeno, S.; Tsujii, Y.; Fukuda, T. Suspensions of Silica Particles Grafted with Concentrated Polymer 
Brush: Effects of Graft Chain Length on Brush Layer Thickness and Colloidal Crystallization. Macromolecules 2007, 40 (25), 9143-9150.

(22) Koerner, H.; Drummy, L. F.; Benicewicz, B.; Li, Y.; Vaia, R. A. Nonisotropic Self-Organization of Single-Component Hairy Nanoparticle Assemblies. ACS Macro Lett. 2013, 2 (8), 670-676.

(23) Jiao, Y.; Tibbits, A.; Gillman, A.; Hsiao, M.-S.; Buskohl, P.; Drummy, L. F.; Vaia, R. A. Deformation Behavior of PolystyreneGrafted Nanoparticle Assemblies with Low Grafting Density. Macromolecules 2018, 51 (18), 7257-7265.

(24) Hansoge, N. K.; Huang, T.; Sinko, R.; Xia, W.; Chen, W.; Keten, S. Materials by Design for Stiff and Tough Hairy Nanoparticle Assemblies. ACS Nano 2018, 12 (8), 7946-7958.

(25) Ethier, J. G.; Hall, L. M. Structure and Entanglement Network of Model Polymer-Grafted Nanoparticle Monolayers. Macromolecules 2018, 51 (23), 9878-9889.

(26) Genix, A. C.; Bocharova, V.; Kisliuk, A.; Carroll, B.; Zhao, S.; Oberdisse, J.; Sokolov, A. P. Enhancing the Mechanical Properties of Glassy Nanocomposites by Tuning Polymer Molecular Weight. ACS Appl. Mater. Interfaces 2018, 10 (39), 33601-33610.

(27) Anderson, J. A.; Lorenz, C. D.; Travesset, A. General purpose molecular dynamics simulations fully implemented on graphics processing units. J. Comput. Phys. 2008, 227 (10), 5342-5359.

(28) Glaser, J.; Nguyen, T. D.; Anderson, J. A.; Lui, P.; Spiga, F.; Millan, J. A.; Morse, D. C.; Glotzer, S. C. Strong scaling of generalpurpose molecular dynamics simulations on GPUs. Comput. Phys. Commun. 2015, 192, 97-107.

(29) Howard, M. P.; Anderson, J. A.; Nikoubashman, A.; Glotzer, S. C.; Panagiotopoulos, A. Z. Efficient neighbor list calculation for molecular simulation of colloidal systems using graphics processing units. Comput. Phys. Commun. 2016, 203, 45-52.

(30) Kremer, K.; Grest, G. S. Dynamics of entangled linear polymer melts: A molecular-dynamics simulation. J. Chem. Phys. 1990, 92 (8), 5057-5086.

(31) Israelachvili, J. N. Intermolecular and surface forces; Academic Press, 2011.

(32) Hui, C. M.; Pietrasik, J.; Schmitt, M.; Mahoney, C.; Choi, J.; Bockstaller, M. R.; Matyjaszewski, K. Surface-Initiated Polymerization as an Enabling Tool for Multifunctional (Nano-)Engineered Hybrid Materials. Chem. Mater. 2014, 26 (1), 745-762.

(33) Humphrey, W.; Dalke, A.; Schulten, K. VMD: Visual molecular dynamics. J. Mol. Graphics 1996, 14 (1), 33-38.

(34) Cang, Y.; Reuss, A. N.; Lee, J.; Yan, J.; Zhang, J.; AlonsoRedondo, E.; Sainidou, R.; Rembert, P.; Matyjaszewski, K.; Bockstaller, M. R.; Fytas, G. Thermomechanical Properties and Glass Dynamics of Polymer-Tethered Colloidal Particles and Films. Macromolecules 2017, 50 (21), 8658-8669.

(35) Gaunaurd, G. C.; Wertman, W. Comparison of effective medium theories for inhomogeneous continua. J. Acoust. Soc. Am. 1989, 85 (2), 541-554.

(36) Zwanzig, R.; Mountain, R. D. High-Frequency Elastic Moduli of Simple Fluids. J. Chem. Phys. 1965, 43 (12), 4464-4471.

(37) Rabani, E.; Egorov, S. A. Integral equation theory for the interactions between passivated nanocrystals in supercritical fluids: Solvophobic and solvophilic cases. J. Phys. Chem. B 2002, 106 (26), 6771-6778.

(38) Mcewan, M. E.; Egorov, S. A.; Ilavsky, J.; Green, D. L.; Yang, Y. Mechanical reinforcement of polymer nanocomposites: theory and ultra-small angle X-ray scattering (USAXS) studies. Soft Matter 2011, 7 (6), 2725-2733.

(39) Mirigian, S.; Schweizer, K. S. Elastically cooperative activated barrier hopping theory of relaxation in viscous fluids. II. Thermal liquids. J. Chem. Phys. 2014, 140 (19), 194507.

(40) Snook, I. K.; Henderson, D. Monte Carlo study of a hardsphere fluid near a hard wall. J. Chem. Phys. 1978, 68 (5), 2134-2139.

(41) Choi, J.; Hui, C. M.; Pietrasik, J.; Dong, H.; Matyjaszewski, K.; Bockstaller, M. R. Toughening fragile matter: mechanical properties of particle solids assembled from polymer-grafted hybrid particles synthesized by ATRP. Soft Matter 2012, 8 (15), 4072-4082.
(42) Schmitt, M.; Choi, J.; Min Hui, C.; Chen, B.; Korkmaz, E.; Yan, J.; Margel, S.; Burak Ozdoganlar, O.; Matyjaszewski, K.; Bockstaller, M. R. Processing fragile matter: effect of polymer graft modification on the mechanical properties and processibility of (nano-) particulate solids. Soft Matter 2016, 12 (15), 3527-3537.

(43) Kröger, M. Shortest multiple disconnected path for the analysis of entanglements in two-and three-dimensional polymeric systems. Comput. Phys. Commun. 2005, 168 (3), 209-232.

(44) Karayiannis, N.; Kroger, M. Combined molecular algorithms for the generation, equilibration and topological analysis of entangled polymers: methodology and performance. Int. J. Mol. Sci. 2009, 10 (11), 5054-5089. 